\title{
Fibre laser torsion sensor system using an excessively tilted fibre grating and low-cost time domain demodulation
}

\author{
Chengbo Mou, Kaiming Zhou, Rui Suo, Lin Zhang, and Ian Bennion \\ Photonics Research Group, Aston University, Birmingham, UK, B4 7ET
}

\begin{abstract}
In this paper, we report a simple fibre laser torsion sensor system using an intracavity tilted fibre grating as a torsion encoded loss filter. When the grating is subjected to twist, it induces loss to the cavity, thus affecting the laser oscillation build-up time. By measuring the build-up time, both twist direction and angle on the grating can be monitored. Using a low-cost photodiode and a two-channel digital oscilloscope, we have characterised the torsion sensing capability of this fibre laser system and obtained a torsion sensitivity of $\sim 412 \mu \mathrm{s} /(\mathrm{rad} / \mathrm{m})$ in the dynamic range from $-150^{\circ}$ to $+150^{\circ}$.
\end{abstract}

Key words: Torsion sensor, fibre laser, tilted fibre grating

\section{INTRODUCTION}

Embedded torsion sensors are important devices to monitor the health condition of engineering structures and have been received considerable attention for smart structure applications. Traditional torsion sensors are based on angle measurement devices like optical encoders and magnetic sensors ${ }^{1}$. Optical fibre grating based torsion sensors are intrinsically small and easy to implement. A corrugated long period grating (LPG) has been demonstrated for torsion sensing ${ }^{2}$. Unfortunately, this kind of sensor can not address the twist direction. Wang and Rao proposed a $\mathrm{CO}_{2}$ laser fabricated LPG based torsion sensor which can measure both twist angle and direction ${ }^{3}$. Tilted fibre grating using polarisation properties has also been described as a passive torsion sensor with capability measuring both twist direction and rate ${ }^{4}$. However, so far, the reported optical torsion sensors are all passive and need expensive optical spectrum analyser and external broadband source to demodulate the optical signal. In this report, we present the first low-cost time domain measurement based torsion sensor using an excessively tilted fibre grating (ETFG) incorporated in a fibre laser cavity. By measuring the fibre laser build-up time using a low cost photodiode and a standard oscilloscope, both twist direction and angle can be obtained simply.

\section{EXPERIMENTAL SET UPAND OPERATION PRINCIPLE}

\subsection{Fabrication of ETFG}

For this experiment, a normal structure fibre Bragg grating (FBG) and a ETFG with fringe structure tiled at $79^{\circ}\left(79^{\circ}\right.$-ETFG) were inscribed in $\mathrm{H}_{2}$-loaded standard telecom fibre (SMF-28) using 244nm UV beam from a frequency doubled Argon ion laser and the standard scanning mask technique. The amplitude mask (Edmund Optics) used for fabricating the ETFG having a period of $6.6 \mu \mathrm{m}$ was rotated by $73^{\circ}$ in respect to the fibre axis in the UV-inscription to generate index fringes at $79^{\circ}$ in the fibre core. A phase mask with a period of $1.07 \mu \mathrm{m}$ was used to UV-inscribe the normal FBG with a reflectivity of $\sim 6 \mathrm{~dB}$ at $1551 \mathrm{~nm}$. As our previous work reported that ETFGs exhibit strong polarisation mode split property ${ }^{5}$, the fabricated $79^{\circ}$-ETFG has shown pronounced dual-peak feature on its transmission spectrum, induced by the polarisation

20th International Conference on Optical Fibre Sensors, edited by Julian Jones, Brian Culshaw, Wolfgang Ecke, José Miguel López-Higuera, Reinhardt Willsch, Proc. of SPIE Vol. 7503, 75033E (c) 2009 SPIE $\cdot$ CCC code: $0277-786$ X/09/\$18 $\cdot$ doi: 10.1117/12.833595

Proc. of SPIE Vol. 7503 75033E-1 
mode split. Fig. 1 shows the transmission spectra of one paired polarisation loss peaks of the ETFG for the probe light at three different polarisation states and inset shows the transmission interchange between the split peaks when the polarisation state changes.

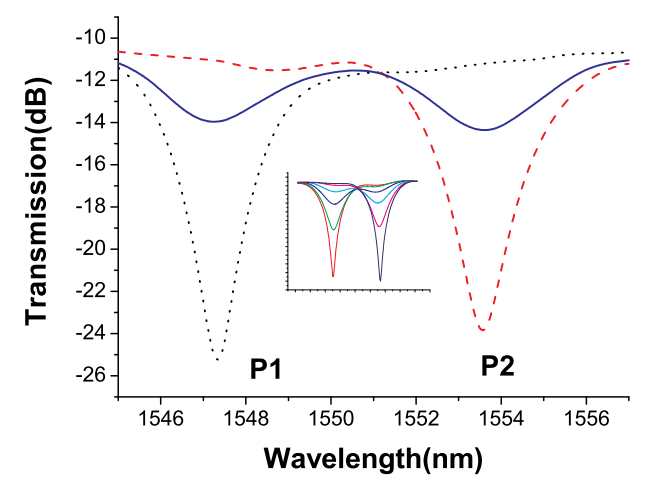

Figure.1. Spectra of the $79^{\circ}$-ETFG probed by unpolarised light (solid line) and polarised light at two orthogonal states (dotted line and dashed line). Inset: the transmission interchange between the split peaks when the polarisation state changes.

\subsection{ETFG based Fibre Laser Torsion Sensor System}

The set-up for the proposed ETFG based fibre laser torsion sensor system is shown in Fig.2 (a). In this configuration, the gain medium is a $\sim 1 \mathrm{~m}$ Erbium/Ytterbium co-doped fibre, which is pumped by a $975 \mathrm{~nm}$ laser diode through a 980/1550 WDM coupler. A $3 \mathrm{~dB}$ coupler formed sagnac loop is employed as the high reflection mirror. The pump is modulated by a square wave through a standard function generator at $5 \mathrm{~Hz}$. The laser output is connected to a low noise photodiode, and the build-up time is measured via a two-channel digital oscilloscope (Tektronix TDS2012).

(a)

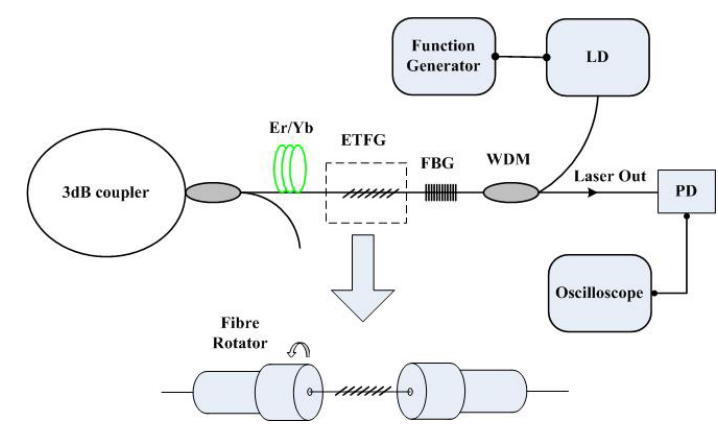

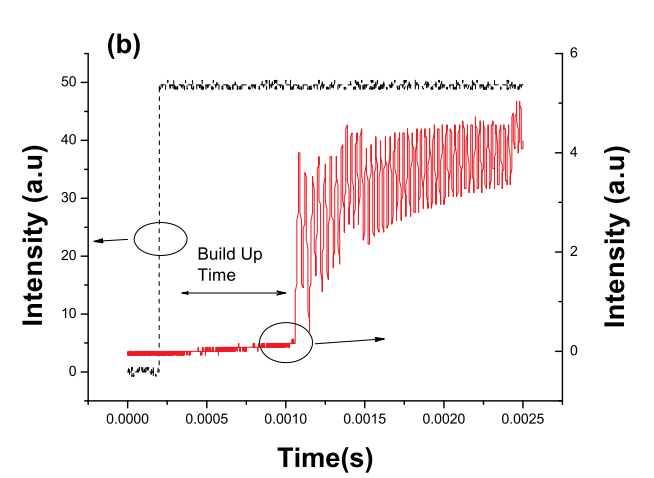

Figure.2 (a) Schematic diagram of the propose ETFG based fibre laser torsion sensor system. (b) Typical build-up time trace of the fibre laser observed on a digital oscilloscope. Modulation signal is shown in dotted line; laser output signal is shown in solid line.

\subsection{Torsion Sensing Principle}

In a modulated laser system, when the pump is switched on, the laser will reach a stable state after a series of relaxation oscillations. Thus, there is a finite build-up time associated with each lasing state at certain pump and loss conditions. Therefore, if the gain medium and the pump condition are not changing, the laser build-up time is only related to the intracavity loss. Because the ETFG itself is a polarisation dependent loss filter, when an ETFG is inserted to the laser cavity 
and subject to twist, the intracavity loss will change, then affecting the laser build-up time. Based on this principle, the torsion experienced by the grating can be monitored by measuring the build-up time of the laser cavity. Fig.2 (b) shows a typical oscillation trace of the laser oscillation build-up process.

\section{EXPERIMENTAL RESULTS}

In the experiment, a segment of laser cavity fibre with an UV-inscribed $\sim 12 \mathrm{~mm} 79^{\circ}$-ETFG was fixed by a clamp on one side and the other side was mounted on a fibre rotator, as shown in Fig. 2 (a). The fibre was under a little tension to position fibre straight in order to eliminate the crosstalk induced from other effects such as axial strain, bending etc. In the experiment, the low and up modulation pump levels were first set at constant values of $14.6 \mathrm{~mW}$ and $43.3 \mathrm{~mW}$, respectively, and the clamped fibre was twisted from 0 to $150^{\circ}$ with an increment of $10^{\circ}$ in both clockwise and anti-clockwise directions. The build-up time was measured for each twist and the normalised results are plotted in Fig. 3 (a). From the figure we can see clearly that at the initial position, i.e. under $0^{\circ}$ twist, the ETFG polarisation loss is at maximum, so the build-up time is at largest value and when the fibre is twisted in clockwise and anti-clockwise direction, the polarisation loss decreases and the build-up time reduces accordingly with increasing twist. This twist experiment was repeated for a different low pump modulation level set at $24 \mathrm{~mW}$ and we saw that the overall torsion sensitivity was decreased for this low pump modulation level, as the lower trace shown in Fig. 3 (a). For a better interpretation of the results, we re-plot the results for twist applied in clockwise and anti-clockwise directions separately in Fig. 3 (b) and (c). From these two figures we can see the system gives a near-linear response in the dynamic range $\pm 140^{\circ}$, giving an estimated torsion sensitivity of $\sim 412 \mu \mathrm{s} /(\mathrm{rad} / \mathrm{m})$. More importantly, we see if the system is set at defined position, i.e. at $\pm 80^{\circ}$ as shown in Fig. 3 (b) and (c), the twist direction can be easily identified.

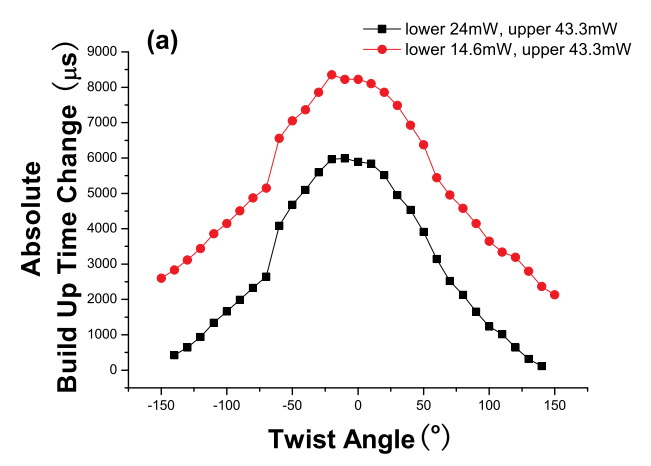



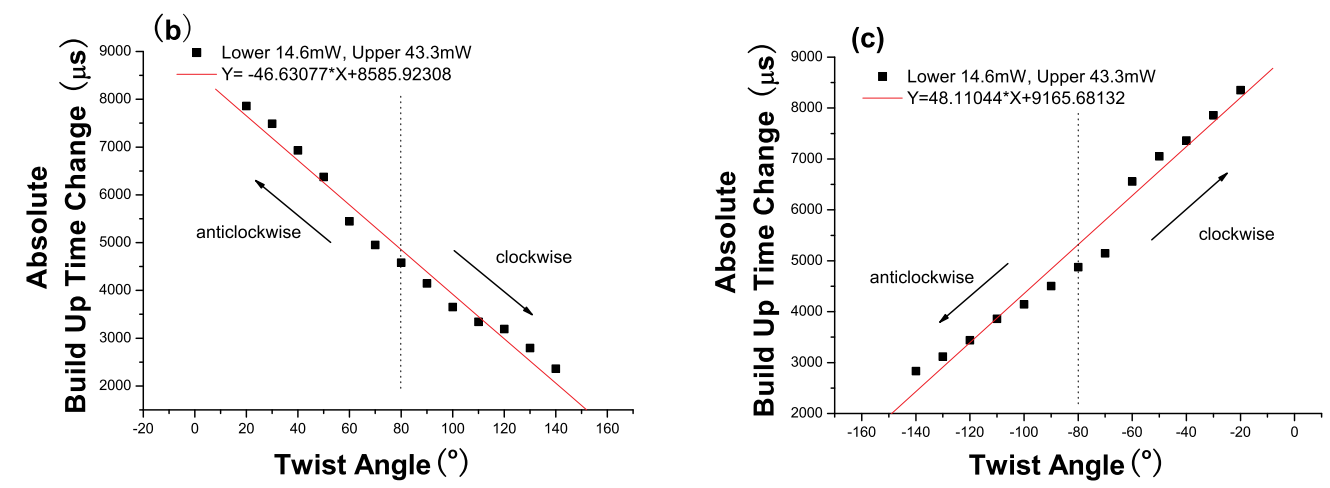

Figure.3 (a) Laser oscillation build-up time against twist angle for twist applied to clockwise and anti-clockwise directions for two different low modulation pump power levels, and re-plotted separately for (b) clockwise and (c) anti-clockwise direction to show the capability of identifying twist direction.

\section{DISCUSSION AND CONCLUSION}

In summary, we have presented a torsion sensor capable of sensing twist angle and direction based on the measurement of transient time of a simple fibre laser incorporating an intracavity ETFG. Within this scheme, the twist applied on the ETFG changes the cavity loss, which alters the laser build-up time. Since the torsion sensing signal demodulation is translated into a time domain regime, only low cost photodiode and standard oscilloscope are required for signal demodulation. This makes the proposed ETFG based fibre laser torsion sensor system simple to use and very cost effective.

\section{ACKNOWLEDGEMENT}

The authors would like to thank Dr. Xuewen Shu for providing the laser gain medium.

\section{REFERENCE}

1. Lemarquand, V, "Synthesis study of magnetic torque sensors", IEEE Trans. Magn., 35,4503-4510, 1999

2. L A Wang, C Y Lin and G W Chern,"A torsion sensor made of a corrugate long period grating", Meas.Sci.Technol. 12, 793-799, 2001

3. Y. P. Wang and Y. J. Rao, "Long period fibre grating torsion sensor measuring twist rate and determining twist direction simultaneously," Electron. Lett., 40, 164-166, 2004.

4. X.Chen, K.Zhou, Lin Zhang and Ian Bennion, "in-fiber twist sensor based on a fibre bragg grating with 81 tilted structure," 18, 2596-2598, 2006

5. Kaiming Zhou, Lin Zhang, Xianfeng Chen, and Ian Bennion, "Optic sensors of high refractive-index responsivity and low thermal cross sensitivity that use fiber Bragg gratings of $>80^{\circ}$ tilted structures," 31,1193-1195,2006 